\title{
Wann gehören Partiturtexte zum ,Werkganzen` eines Opernlibrettos?
}

Dem Opernlibretto ${ }^{1}$ kommt seit einigen Jahrzehnten sowohl von musik- als auch von literaturwissenschaftlicher Seite immer mehr Aufmerksamkeit zu. Aber in bestimmten rein ontologischen Grundfragen scheint Unsicherheit zu herrschen, eine Unsicherheit, die sich auch auf die editionspraktische und -theoretische Beschäftigung mit dem Problembereich auswirkt. Hierbei spielt nicht nur die fachlich-disziplinäre Vielfalt und Widersprüchlichkeit in dem aus Musikwissenschaft und (mehreren) Literaturwissenschaften bestehenden Feld eine Rolle, sondern zugegebenermaßen auch die Komplexität des Gegenstandes selbst, die allzu pauschale Urteile verbietet. Trotz dieser Feststellung sind wichtige Ansätze eines Dialogs zwischen Germanistik und Musikwissenschaft, die sich unter anderem im nach wie vor sehr nützlichen Sammelband Der Text im musikalischen Werk von 1998 schriftlich niedergeschlagen hat, ${ }^{2}$ hervorzuheben.

Geprägt wird der Diskurs zum Libretto vielfach immer noch durch eine Auffassung von der Vertonung, nach der diese (und zwar unabhängig von der Entstehungsgeschichte) irgendeine Art Decodierung darstelle, durch die allein das Libretto beurteilt und analysiert werden könne. ${ }^{3}$ Grundsätzlich richtiger erscheint dabei die

\footnotetext{
${ }^{1}$ Auch Texte zu Oratorien, Kantaten und Balletten werden häufig als Libretti bezeichnet. Wenn im Folgenden von Libretti die Rede ist, ist der Terminus jedoch auf Opernlibretti bezogen. Dies verhindert an sich nicht, dass mehrere Schlussfolgerungen auch für die (vor allem germanistische) Edition von vor allem Oratorien- und Kantatenlibretti zutreffen könnten.

${ }_{2}^{2}$ Der Text im musikalischen Werk. Editionsprobleme aus musikwissenschaftlicher und literaturwissenschaftlicher Sicht. Hrsg. von Walther Dürr u.a. Berlin 1998 (Beihefte zur Zeitschrift für deutsche Philologie. 8). Vgl. auch eine frühere italienische Entsprechung: L'edizione critica tra testo musicale e testo letterario. Atti del convegno internazionale (Cremona 4-8 ottobre 1992). Hrsg. von Renato Borghi und Pietro Zappalà. Lucca 1995 (Studi e testi Musicali. Nuova Serie. 3).

${ }^{3}$ In Antje Tumats Ausführungen zum Libretto im Allgemeinen (als ,Textsorte') wird diese Sichtweise mehrmals augenfällig, so wenn sie Albert Gier methodische Unvollständigkeit vorwirft, und zwar weil Giers Konzept der „Vertonbarkeit“ „gänzlich ohne Betrachtung der tatsächlichen Vertonung, also der Musik, verhandelt“ werde (Antje Tumat: Dichterin und Komponist: Ästhetik und Dramaturgie in Ingeborg Bachmanns und Hans Werner Henzes „Prinz von Homburg“. Kassel 2004, S. 19). Aus einer entstehungsgeschichtlichen, editorischen Perspektive sollte jedoch deutlich sein, dass sich Vertonbarkeit und tatsächliche Vertonung auf zwei verschiedenen Stufen im Entstehungs- und Bearbeitungsprozess eines Textes befinden. Auch später (vgl. z.B. Tumat 2004, S. 26) ist zu fragen, von welcher Ebene Tumats Kritik an Giers Textverständnis ausgeht. Tumat behauptet vom Libretto ferner, ein solches könne „letztlich nur als Teil der Oper, also interdisziplinär, bewertet werden, da es für seine Funktion innerhalb des Gesamtereignisses Oper konzipiert ist“ (Tumat 2004, S. 10), eine Argumentation, in der ebenfalls zwischen einer textuellen Eigenschaft auf der Ebene der Konzeption und einer späteren kompositorischen Umsetzung nicht deutlich unterschieden wird: Die Vertonung wird quasi als die Decodierung des Librettos verstanden. Im musikeditorischen Kontext fragt Ulrich Konrad in noch problematischerem Duktus, „wie sinnvoll die isolierte Edition von Libretti ist [...], eine Edition, welche die Autonomie des Wortkunstwerks dort beansprucht, wo die Funktion der Dichtung als Vorlage für musikalische Adaption konstitutiv ist" (Ulrich Konrad: Der Bürger als Edelmann und Ariadne auf Naxos von Hugo von Hofmannsthal und Richard Strauss: Editionsprobleme bei einer „sehr ernsthaften Spielerei“. In: Opernedition.
} 
Feststellung des Literaturwissenschaftlers Albert Gier: „Wenn ein Komponist ein Libretto vertont, deutet er es auf individuelle Weise aus; seine Interpretation kann nur eine von zahlreichen (oder zahllosen) möglichen sein“. ${ }^{4}$ Die Tatsache, dass viele - auch deutschsprachige $^{5}$ - Libretti des 18. Jahrhunderts mehrfach vertont wurden, ist nur ein Argument unter mehreren für diese Schlussfolgerung. Die Musikwissenschaftlerin Helga Lühning beschreibt das Libretto als „Vorlage“ und „sprachliche Autorität“ für die Vertonung: Sie stellt fest, dass „das Libretto die Basis der Komposition bildet, daß es sie anregt, ihr Bedingungen stellt, sie vorausprägt “. ${ }^{6}$ Es sollte jedoch hinzugefügt werden, dass derartige Feststellungen durch eine konsequent textgeschichtlich orientierte und editionswissenschaftliche Perspektive erheblich erleichtert werden, gleichzeitig aber differenziert werden müssen.

Eine germanistische Editionstheorie des Librettos ist deswegen nicht nur für die Editionspraxis (in erster Linie innerhalb der Germanistik und mit gewissen Vorbehalten auch für andere Literaturwissenschaften, vielleicht auch für die Musikwissenschaft), sondern auch für die allgemeine Gattungstheorie des Librettos von Bedeutung. In meinem laufenden, vom schwedischen Forschungsrat Vetenskapsrådet finanzierten Projekt zur Editionstheorie des Librettos untersuche ich zwei größere Problembereiche: erstens die Abgrenzung des ,Werkganzen ${ }^{7}$ bei einem Libretto und zweitens Probleme, die bei Vergleichen, etwa in Variantendarstellungen, zwischen Literaturtexten und Partiturtexten ${ }^{8}$ auftreten. Im Rahmen des vorliegenden Beitrags geht es ausschließlich um die erste Frage, um das ,Werkganze` des Librettos. Es werden im Folgenden einige noch vorläufige Überlegungen zum Thema vorgestellt; eine definitivere und vollständigere Darstellung der Problematik, auch im Kontext der übergreifenden Theorie, ist für eine spätere Monografie vorgesehen.

Die Diskussion um das Werkganze der Oper ist aus dem musikeditorischen Diskurs bekannt, und diese Überlegungen, wie die von Helga Lühning zum zweifelhaften

Bericht über das Symposion zum 60. Geburtstag von Sieghart Döring. Hrsg. von Helga Lühning und Reinhard Wiesend. Mainz 2005, S. 161-177, hier S. 171f.). Gerade die Entstehung als Vorlage für eine spätere Vertonung sollte umgekehrt deutlich machen, dass die Kompositionsvorlage textgeschichtlich gesehen in der Regel einen von dieser späteren Interpretation losgelösten und eigenständigen Status hat.

${ }^{4}$ Albert Gier: Das Libretto: Theorie und Geschichte einer musikoliterarischen Gattung. Darmstadt 1998, S. 17.

${ }^{5} \mathrm{Im}$ Folgenden werden in erster Linie, der germanistischen Ausrichtung meines Projekts entsprechend, deutschsprachige Beispiele herangezogen.

${ }^{6}$ Helga Lühning: Die Texte des Fidelio. In: Leonore. Oper in zwei Aufzügen von Ludwig van Beethoven. Das Libretto der Aufführung von 1806. Hrsg. von Helga Lühning. Bonn 1996, S. 3-25, hier S. 20.

${ }^{7}$ Eine kurze definitorische Diskussion zu diesem zumindest in der Germanistik sehr umstrittenen und seit langem von vielen für obsolet gehaltenen Begriff ,Werk‘ (vgl. zur Diskussion zuletzt Klaus Hurlebusch: Steckt für Quellen- und Editionsphilologen nur der liebe Gott im Detail? In: editio 25, 2011, S. 1-31) folgt unten.

${ }^{8}$ Definitionen siehe weiter unten. 
Stellenwert der Dialoge in der Edition einer Opernpartitur, ${ }^{9}$ sind $\mathrm{zu}$ beachten; eine germanistische Editionstheorie hat jedoch die umgekehrte Perspektive, hat also nicht die Oper als Werk, sondern das Libretto als Werk und dessen Abgrenzung zu untersuchen. Aus jenem germanistischen, editionswissenschaftlichen Ausgangspunkt stellt sich daher eine andere Grundfrage, und zwar vor dem Hintergrund, dass das Libretto häufig in zwei voneinander grundsätzlich verschiedenen schriftlichen Erscheinungsformen auftritt, einerseits als separater verbalsprachlicher Text und andererseits als den Noten unterlegter Text in einer Partitur oder einem Klavierauszug. Diese von anderen literarischen Gattungen abweichende Überlieferung motiviert m.E. eine gattungseigene Editionstheorie des Opernlibrettos. Damit wird die prinzipielle Gattungsunabhängigkeit der Edition und Editionstheorie, so wie sie 1989 von Siegfried Scheibe behauptet wurde, in Frage gestellt. ${ }^{10}$ Das Phänomen der Partiturtexte und die mögliche Zusammenarbeit zwischen Librettist und Komponist stellen den germanistischen Editor eines Librettos vor besondere Herausforderungen. Auf gewisse theoretisch wie praktisch bedingte Probleme wird er immer stoßen - etwa: Wer ist als Autor zu bezeichnen? Wie sind Überlieferung und Textgenese abzugrenzen? Welche Varianten sind als Entstehungsvarianten anzuführen?

Die Fragestellung, der im Folgenden nachzugehen ist, ist an die gerade erwähnten geknüpft, nimmt aber explizit Bezug auf die eine der zwei genannten schriftlichen Erscheinungsformen: Unter welchen Umständen ist es angebracht, den verbalsprachlichen Text einer Partitur, eines Klavierauszugs oder anderer Notentexte zur Textgenese eines Opernlibrettos, zum ,Werkganzen‘ dieses Librettos zu rechnen? Auf der materiellen Ebene wäre die Fragestellung wie folgt zu formulieren: Wann gehören derartige Textträger zur Überlieferung eines Librettos?

An sich betrifft die hier notwendige Unterscheidung nicht nur Texte in Partituren, sondern auch Texte ohne musikalische Notation, die ausschließlich auf den Komponisten zurückgehen und die bei vielen der unten folgenden Überlegungen mitzudenken sind. Im Folgenden wird deswegen in erster Linie von den Texten in Partituren und anderen in musikalischer Notation eingebetteten Texten die Rede sein, weil an dieser Erscheinungsform und deren Besonderheiten mehr prinzipielle Fragestellungen verschiedener Art geknüpft sind.

\footnotetext{
${ }^{9}$ Vgl. Helga Lühning: Gehören Operndialoge in eine „Werk“-Ausgabe? In: Der Text im musikalischen Werk 1998 (Anm. 2), S. 169-183.

${ }^{10}$ Siegfried Scheibe: Benötigen wir eine eigene Theorie der Edition von Dramen? Einige Bemerkungen zur Einheit der Textologie. In: editio 3, 1989, S. 28-40. Zwar kann ich Scheibe in seinem Widerstand gegen eine gesonderte Drameneditionstheorie grundsätzlich Recht geben, doch im Falle des Librettos, das Scheibe dabei nicht bespricht, wird es weitaus problematischer. Scheibe operiert mit den Kriterien Arbeitsweise und Überlieferung, und beide, insbesondere die Überlieferung, sehen bei Libretti in den meisten Fällen anders als bei anderen literarischen Werken aus.
} 
Zwar edieren Philologen seit langem Libretti, nicht zuletzt in Gesamtausgaben, aber auf das theoretische Gebiet haben sich nur wenige Germanisten ${ }^{11}$ und andere Philologen begeben. Für die Germanistik sehr relevante Überlegungen zur Librettoeditionstheorie finden sich aber auch in der Musikwissenschaft, nicht zuletzt innerhalb der Ausgabe der Sämtlichen Werke Wagners und in deren Umkreis: Hier sind Beiträge von Egon Voss, Cristina Urchueguía, Reinhard Strohm ${ }^{12}$ und Werner Breig zu erwähnen.

Gerade Werner Breigs Unterscheidung zwischen ,Literaturtexten“ und ,Partiturtexten ${ }^{13}$ wird für die folgende Diskussion übernommen. Als ,Literaturtext ${ }^{`}$ bezeichne ich jeden Text, der den Werktext eines Librettos ohne Noten enthält; mit ,Partiturtext` ist der verbalsprachliche Text gemeint, der in musikalischer Notation integriert erscheint. Die Termini sind im Kontext der Wagner-Forschung entstanden, und beide tragen Probleme mit sich. Allerdings hat sich bislang keine andere haltbare Terminologie für die betreffenden Gegenstände durchgesetzt. Zum Terminus, Literaturtext ${ }^{\star}$ sollte erstens vermerkt werden, dass dem Glied ,Literatur' hier kein Qualitätsmerkmal zugrunde liegt. Zweitens könnten auch die Partiturtexte in gewisser Weise als literarisch gelten; sie teilen das Wortmaterial häufig mit den Literaturtexten, auch wenn einige Eigenschaften der Letzteren, wie die Verseinteilung (bei Verslibretti), hier nicht vorkommen. Entscheidende Kennzeichen eines Literaturtextes sind, dass dieser in einem rein schriftsprachlichen Kontext vorkommt und eine auffällige Nähe zu einer anderen literarischen Form, dem Sprechdramentext, aufweist. ,Partiturtexte“ sind an sich nicht an die Form der Orchesterpartitur gebunden, sondern können sich ebenso gut in Kompositionsskizzen, Particellen, Klavierauszügen und Chorstimmen finden.

Die Mehrdeutigkeit und die begriffliche Problematik in Bezug auf den Terminus ,Libretto“ sind bekannt. Albert Gier stellt fest, dass „,[d]as Libretto [...] sowohl Text [...] wie

\footnotetext{
${ }^{11}$ Vgl. Dirk Hoffmann: Das dynamische Textverständnis - die Basis der kritischen Edition des „Rosenkavalier““ In: editio 3, 1989, S. 130-144: Bodo Plachta: Libretti: eine von den Editoren vergessene Gattung? Überlegungen zur kommentierenden Herausgabe von Operntextbüchern des 17 . und 18. Jahrhunderts. In: Kommentierungsverfahren und Kommentarformen: Hamburger Kolloquium der Arbeitsgemeinschaft für germanistische Edition 4. bis 7. März 1992, autor- und problembezogene Referate. Hrsg. von Gunter Martens. Tübingen 1993 (Beihefte zu editio. 5), S. 25-37; Bodo Plachta: Zwischen Musiktheater und Sprechtheater: Zur literaturwissenschaftlichen Begründung einer Edition deutschsprachiger Operntexte des 17. und 18. Jahrhunderts. In: Der Text im musikalischen Werk 1998 (Anm. 2), S. 147-156; und mehr am Rande KlausDieter Krabiel: Die Lehrstücke Brechts als editorisches Problem. In: Der Text im musikalischen Werk 1998 (Anm. 2), S. 331-345.

12 Strohms Beitrag zur Librettoeditionstheorie ist hier besonders hervorzuheben; er weist auf Funktionsdifferenzen und typische Textvarianten zwischen Partitur und Libretto (Literaturtext) hin: Reinhard Strohm: Partitur und Libretto: Zur Edition von Operntexten. In: Opernedition 2005 (Anm. 3), S. 37-56, bes. S. 55.

${ }^{13}$ Werner Breig: Überlegungen zur Edition von Richard Wagners musikdramatischen Texten. In: Der Text im musikalischen Werk 1998 (Anm. 2), S. 284-311, hier S. 286.
} 
$B u c h^{\text {“14 }}$ sei, und Cristina Urchueguía unterscheidet zwischen drei Bedeutungen des Wortes: einer „textliche[n] Vorlage“, dem ,als Oper realisierten Text" und auch einer Publikationsform. ${ }^{15}$ Als Opernlibretto bezeichne ich im Folgenden einen verbalsprachlichen dramatischen (schriftlich überlieferten) Text, der für eine Vertonung (zur Oper) vorgesehen oder in dieser Funktion bereits umgesetzt worden ist. Das Libretto kann in der Form von Literaturtexten für sich allein stehend, aber auch in einen anderen Kontext, den der musikalischen Notation, integriert erscheinen: in Partiturtexten. Für die Publikationsform ist derselbe Terminus dagegen nicht zu benutzen: Diese bezeichne ich als ,Textbuch` oder, präziser ausgedrückt, als ,gedruckten Literaturtext‘.

Angesichts dieser Definition wird auch deutlich, dass eine Differenzierung hinsichtlich der Funktionen von solchen Texten notwendig ist: Ein (Literatur-)Text kann sehr wohl eine Funktion als Kompositionsvorlage haben, und deren Entstehung vor der Vertonung ist auch ein wichtiges Argument für die grundsätzliche Autonomie des Librettos; es kann sich aber auch um einen verbalsprachlichen Text handeln, der, bereits vertont, in eine musikalische Notation eingebettet ist (Partiturtext), oder um einen, der wiederum als - vielleicht gedruckter - Literaturtext den Prozess der Vertonung und die dabei entstandenen Änderungen bereits widerspiegelt und diese eingearbeitet hat. Auch Literaturtexte, die zwar nach der Vertonung entstehen, aber ausschließlich auf die Kompositionsvorlage, nicht auf den vertonten Text, zurückgehen, sind noch als Libretti zu bezeichnen.

So wie ich im Folgenden, aber mit anderen Argumenten und mehr Vorbehalten, schreibt auch Reinhard Strohm dem Libretto unter Umständen ,Werkstatus ${ }^{6}$ zu. $^{16}$ Das ist für eine Editionstheorie des Librettos an sich kein erforderliches Merkmal, denn Edierbarkeit und Werkstatus sind bekanntlich zwei verschiedene Dinge; schließlich kann der Germanist auch Briefe, private Tagebücher, Notizbücher usw. edieren. Der Begriff ,Werk' ist im Folgenden als Bezeichnung für die Gesamtheit der textuellen Spuren einer Arbeit an einem gewissen Wortmaterial literarischen Charakters zu verstehen. Die Zugehörigkeit einer Anzahl von Texten zu einem Werk ist m.E. dadurch zu erkennen, dass sie von einem Autor bzw. einer Gruppe von zusammenarbeitenden Autoren stammt oder autorisiert ist, ferner durch einen

\footnotetext{
${ }^{14}$ Gier 1998 (Anm. 4), S. 3 (Kursive im Original).

${ }^{15}$ Cristina Urchueguía: Richard Wagners plurale Autorschaft: Überlegungen zur Edition von Richard Wagners Libretti am Beispiel von Tannhäuser. In: Autor - Autorisation - Authentizität. Beiträge der Internationalen Fachtagung der Arbeitsgemeinschaft für germanistische Edition in Verbindung mit der Arbeitsgemeinschaft philosophischer Editionen und der Fachgruppe Freie Forschungsinstitute in der Gesellschaft für Musikforschung, Aachen, 20. bis 23. Februar 2002. Hrsg. von Thomas Bein u.a. Tübingen 2004 (Beihefte zu editio. 21), S. 293306, hier S. 294.

${ }^{16}$ Strohm schreibt vor allem ,ältere[n]“ Libretti Werkstatus zu, und zwar wegen ,ihrer Fähigkeit, auch ohne Musik konsumierbar zu sein“" (Strohm 2005, Anm. 12, S. 45).
} 
hohen Grad an gemeinsamem Wortmaterial bzw. syntaktischer Beziehbarkeit ausgezeichnet ist. $^{17}$

Die Textgenese und die Überlieferung eines Librettos als literarisches Werk sind in etlichen Fällen mit denen der Oper als musikalisches bzw. plurimediales Kunstwerk eng verflochten, und die Textgenese bzw. das Werkganze des Librettos besteht - so viel sei schon hier gesagt - in den meisten dieser Fälle sowohl aus Literaturtexten als auch Partiturtexten. Letztere können für die Textgenese eines Librettos eine zentrale Bedeutung haben. Meine frühere detaillierte Untersuchung der Textgenese des Librettos Aufstieg und Fall der Stadt Mahagonny von Brecht und Weill hat zum Beispiel erwiesen, dass die Textfassungen sämtlicher späterer Literaturtextträger vom Partiturautograph Kurt Weills stemmatisch abhängig sind. ${ }^{18}$ Ein derartiges Verhältnis ist jedoch keineswegs die Regel.

Das bisher Gesagte betrifft nur die Frage der Zugehörigkeit zur Überlieferung und zum Werkganzen, nicht aber die Frage der Textgrundlage. Dass ein Partiturtext nicht als Textgrundlage für eine Literaturtextausgabe dienen sollte, hat die Wagner-Philologie mit sehr überzeugenden Argumenten untermauert. So schreibt Breig, durch die in vielen Leseausgaben (Stichwort: Reclam) häufig vorkommende Literarisierung des Partiturtextes werde „eine Textgestalt kreiert [...], die für den Autor in dieser Form nicht existierte“. ${ }^{19}$ Voss macht auf die ohne musikalische Notation nicht darstellbaren Transformationen des Textes durch die Musik, vor allem was den Rhythmus betrifft, aufmerksam, ohne die eine Wiedergabe des vertonten Textes sehr problematisch ist. Laut Voss „ignoriert“ ein solches Verfahren außerdem, „dass das Textbuch eine eigene Spezies darstellt““ ${ }^{20}$ Urchueguía bemerkt sehr treffend, dass aus einer Partitur editorisch abstrahierte Partiturtexte bzw. Partiturtextteile auch „einen Teil ihrer semiotischen Bedeutung ein[büßen]“, ${ }^{21}$ wenn sie ohne musikalische Notation erscheinen. Partiturtexte in den Literaturtextmodus zu übertragen ist, wie diese Anmerkungen zeigen, ein editorisch sehr zweifelhafter Vorgang, von dem nur abgeraten werden kann. ${ }^{22}$

\footnotetext{
${ }^{17}$ Dies ist also in erster Linie eine Bestimmung der Zugehörigkeit verschiedener Texte zu einem Werk, keine endgültige oder vollständige Definition des Werkbegriffs. Vgl. zu den Ausgangspunkten Siegfried Scheibe: Werk und Edition: Aus dem Eröffnungsreferat zum „Internationalen Editionskolloquium Berlin 1989“. In: Zu Werk und Text: Beiträge zur Textologie. Hrsg. von dems. und Christel Laufer. Berlin 1991, S. 11-22, hier S. 13; Esbjörn Nyström: Libretto im Progress: Brechts und Weills Aufstieg und Fall der Stadt Mahagonny aus textgeschichtlicher Sicht. Bern u.a. 2005 (Arbeiten zur Editionswissenschaft. 6), S. 71f.

${ }^{18}$ Vgl. Nyström 2005 (Anm. 17), S. 633 und Stemma (Faltblatt).

${ }^{19}$ Breig 1998 (Anm. 13), S. 294.

${ }^{20}$ Vgl. Egon Voss: Nachwort. In: Richard Wagner: Der Ring des Nibelungen. Ein Bühnenfestspiel für drei Tage und einen Vorabend. Erster Tag: Die Walküre. Textbuch mit Varianten der Partitur. Hrsg. von Egon Voss. Stuttgart 1997, S. 113-128, hier S. 126.

${ }^{21}$ Urchueguía 2004 (Anm. 15), S. 296.

${ }^{22}$ Im Anschluss an diese Problematik stellt sich auch die Frage, wie Varianten zwischen Partiturtexten und Literaturtexten wiederzugeben sind und was in diesem Kontext überhaupt als eine Variante zu bezeichnen ist.
} 
Wenn ein Libretto ausschließlich in der Form von Partiturtexten überliefert ist, liegt ein Sonderfall vor - hier ist vorläufig auf die Untersuchungen von Reinmar Emans zu Oratorien- und Kantatenlibretti hinzuweisen. ${ }^{23}$ In solchen Fällen können Partiturtexte in editorischer Hinsicht als Stellvertreter dienen, was allerdings immer noch mit den oben angesprochenen Problemen verbunden ist.

Die Frage nach dem Stellenwert von Partiturtexten in verschiedenen Fällen soll anhand dreier Kriterien beantwortet werden, die in einem gewissen Abhängigkeitsverhältnis voneinander stehen:

- Autorschaftsbezogenes Kriterium: Ist der Komponist als (Mit-)Autor des verbalsprachlichen Textes zu verstehen oder nicht?

- Autorisationsbezogenes Kriterium: Ist der Text durch den Autor/die Autoren autorisiert oder nicht?

- Stemmatisches Kriterium: Sind spätere autorisierte Literaturtexte direkt oder indirekt und hauptsächlich oder zusätzlich von diesem Partiturtext abhängig oder nicht?

Von den so genannten Dichter-Komponisten abgesehen, ist es historisch gesehen eher selten, dass der Komponist in entsprechenden Titeleien als Autor oder Mitautor am Text genannt wird. In diesen Fällen, etwa György Ligeti als Mitlibrettist Michael Meschkes bei Le Grand Macabre, ist ein Kriterium zur Bestimmung der Autorschaft erfüllt. Eine Mitautorschaft des Komponisten kann jedoch viel öfter festgestellt werden. Dabei schließe ich mich den Überlegungen Richard Sperls zur Marx-Engels-Gesamtausgabe an: Entweder wird die Bestimmung einer Doppelautorschaft durch eigene Aussagen der Beteiligten ermöglicht, oder aber es lassen sich „am Text konkrete Anteile des einen oder anderen fest[]machen“; ,[i]n allen anderen Fällen erscheint nur ein Autor“، ${ }^{24}$

Der Einwand liegt nahe, dass der Komponist einer Oper ja zweifellos immer eine Arbeit am verbalsprachlichen Text ausführt. Nicht offensichtlich ist dagegen, ob er in Bezug auf das Libretto als Mitautor oder aber als Textbearbeiter zu bezeichnen ist. Dabei ist das Augenmerk zuerst auf die Beteiligung an der Kompositionsvorlage zu richten, quasi unabhängig von der eigentlichen Kompositionsarbeit. Der Begriff ,Mitautor` weist jedoch

Dass in solchen Fällen keine synoptische, vollständige Wiedergabe von Partiturtext (losgelöst von der musikalischen Notation) und Literaturtext wünschenswert ist, versteht sich schon aus der obigen Diskussion. In weiteren Publikationen innerhalb meines laufenden Forschungsprojekts werde ich zu dieser Fragestellung zurückkehren.

${ }^{23}$ Vgl. Reinmar Emans: Probleme der Textedition bei J.S. Bach. In: Der Text im musikalischen Werk 1998 (Anm. 2), S. 87-97, hier bes. S. 94f.

${ }^{24}$ Richard Sperl: Die Marx-Engels-Gesamtausgabe: Editorische Konsequenzen literarischer Zusammenarbeit zweier Autoren. In: Literarische Zusammenarbeit. Hrsg. von Bodo Plachta. Tübingen 2001, S. 141-155, hier S. 153. 
eine große Spannbreite auf: von der wirklichen, aktiven Zusammenarbeit am Text, wobei sich die Anteile am fertigen Text je 50 \% nähern, der Komponist also einer von zwei ebenbürtigen Autoren wäre, bis hin zu vereinzelten, wenn auch wichtigen Eingriffen während der Entstehung. Im letzteren Fall ist ein hauptsächlicher Autor zu identifizieren, und neben ihm ein Mitautor. Bei qualitativ und quantitativ eher geringfügigen Eingriffen wäre er wohl auch nicht mehr als Mitautor zu betrachten.

Das Kriterium der Autorisation besteht im Folgenden in der Forderung, der Autor bzw. eine Autorengruppe müsse den Text ,zu einem bestimmten Zeitpunkt [...] gebilligt[ [ ] ${ }^{625}$ haben. Bei Gemeinschaftswerken zweier oder mehrerer Autoren, bei denen sich die jeweiligen Anteile deutlich unterscheiden, ist es nicht ohne Relevanz, wer hinter einer einzelnen Autorisierung steht: Ist es ein Mitautor, oder ist es der hauptsächliche Autor? Hat die Zusammenarbeit durch den Tod eines hauptsächlichen Autors aufgehört, muss die Überlieferung m.E. als abgeschlossen gelten; anders ist es dagegen, wenn ein Mitautor gestorben ist.

Das stemmatische Kriterium ist auf die anderen Kriterien und auf andere Bedingungen angewiesen, da die Autorschafts- und Autorisationsverhältnisse zuerst geklärt sein müssen, damit erkennbar ist, welche Literaturtexte überhaupt vom Autor oder von den Autoren autorisiert sind. (Fragezeichen sind hier wegen der Publikationskontexte historisch gesehen häufig bei gedruckten Literaturtexten zu setzen.) Ferner müssen auch die näheren Verwandtschaftsverhältnisse geklärt werden können.

Andererseits ist dieses Kriterium insofern ein wichtiges positives Kriterium, als jede Abhängigkeit eines autorisierten Literaturtextes von einem früheren Partiturtext allein schon über die Zugehörigkeit des betreffenden Partiturtextes zur Textgenese des Librettos positiv entscheidet. Wenn es sich um mehr als nur eine vereinzelte, punktuelle Abhängigkeit handelt, wäre es normalerweise auch angebracht, sämtliche Partiturtexte des betreffenden Komponisten zum Libretto und deren auch interne Verwandtschaftsverhältnisse anzuführen.

Ausschlaggebend für dieses Kriterium ist also, dass Partiturtexte und autorisierte Literaturtexte nicht isoliert voneinander stehen, sondern dass vielmehr von einem Partiturtext eine Deszendenz zur Gruppe der Literaturtexte nachzuweisen ist. Diese Fälle können in der germanistischen Editorik an die Anführung von unautorisierten Drucken erinnern, wenn diese Drucke Deszendenz zu späteren autorisierten Fassungen aufweisen.

\footnotetext{
${ }^{25}$ Zitiert nach einer Standarddefinition in Bodo Plachta: Editionswissenschaft. Eine Einführung in Methode und Praxis der Edition neuerer Texte. 2., ergänzte und aktualisierte Aufl. Stuttgart 2006 [zuerst 1997], S. 135.
} 
Aufgrund der drei genannten Kriterien, des autorschaftsbezogenen, des autorisationsbezogenen und des stemmatischen, kann nun eine Einteilung der in Frage kommenden Texte in vorläufige Kategorien vorgenommen werden, Kategorien, die dann in zwei übergeordnete Gruppen einzuordnen sind: eine Gruppe, in der die Frage nach der Zugehörigkeit der Partiturtexte zur Textgenese eines Librettos negativ, und eine weitere Gruppe, in der sie positiv zu beantworten ist.

Zunächst ist aber eine Ausnahme kurz zu erwähnen: Librettistische Texte, die ganz auf der Grundlage vorhandener Musik entstehen, sind als eine eigene Kategorie zu betrachten, die durch die folgenden Überlegungen nicht abgedeckt wird. $\mathrm{Zu}$ diesen Texten gehören viele Librettoübersetzungen, so etwa Franz Werfels Übersetzungen aus dem Italienischen, aber auch ein ganz andersartiges, rezentes Beispiel wie Irene Disches und Elfriede Jelineks Originallibretto Der tausenjährige [sic] Posten oder Der Germanist (2003), ${ }^{26}$ dessen Gesangstexte auf der Basis von Franz Schuberts Musik zu zwei Singspielen und dabei auch unter weitgehender Übernahme (mit handlungsbedingten Modifizierungen) der bestehenden Texte aus den Libretti entstanden sind. Solche Fälle sind von großem grundsätzlichem Interesse, und ihnen sollte in einem anderen Rahmen besondere Aufmerksamkeit zuteil werden. ${ }^{27}$

Die Kategorien, in denen die Libretti nach den drei oben genannten Kriterien eingeteilt werden können, könnten nach der folgenden Nummerierung 1-10 auch in eine Skala eingeordnet werden. Die Fälle 1 (links) und 10 (rechts) sind dabei die eindeutigsten, die Kategorien um die Mitte dagegen sind Grenzfälle. Es soll hier nicht der Eindruck erweckt werden, als ginge es um 10 quantitativ ungefähr gleich große Gruppen. Einige von ihnen, besonders Gruppe 8, dürften sehr viel häufiger vorkommen als manch andere. Hier folgen die Kategorien:

A. Partiturtexte gehören nicht zur Textgenese des Librettos:

1. Unvertonte Libretti.

2. Libretti, deren Vertonung nach dem Tod des (hauptsächlichen) Autors erfolgt ist.

\footnotetext{
${ }^{26}$ Der Text ist auf der Website www.elfriedejelinek.com zu finden und dort auf 2003 datiert.

27 „Musiktheater ohne Libretto“ (Karin Hochradl: Olga Neuwirths und Elfriede Jelineks gemeinsames Musiktheaterschaffen: Ästhetik, Libretto, Analyse, Rezeption. Bern u.a. 2010, Salzburger Beiträge zur Musikund Tanzforschung. 4, S. 40) wäre ein weiterer Sonderfall, der aber - wenn die Bezeichnung immer wörtlich zu nehmen wäre, was jedoch zweifelhaft erscheint - nicht in den Rahmen einer Librettoeditionstheorie fallen würde. Vgl. die musikeditorischen Überlegungen von Dörte Schmidt: „Texttheater“ oder „Ereignistheater“. Zur Frage der Editionsfähigkeit neuester Opern am Beispiel der Arbeiten von Helmut Lachenmann, Elliott Carter und Meredith Monk. In: Opernedition 2005 (Anm. 3), S. 189-211.
} 
3. Libretti, ohne konkrete Aussicht auf Vertonung entstanden, später vertont; keine stemmatische Verbindung von einem Partiturtext zu einem autorisierten Literaturtext, keine Autorisation eines Partiturtextes durch den Librettisten.

4. Libretti, ohne konkrete Aussicht auf Vertonung durch den betreffenden Komponisten entstanden, später vertont; keine stemmatische Verbindung von einem Partiturtext zu einem autorisierten Literaturtext, keine Autorisation eines Partiturtextes durch den Librettisten.

5. Libretti, nach Vereinbarung, aber ohne Zusammenarbeit mit dem betreffenden Komponisten entstanden (Komponist kein Mitautor), später vertont; keine stemmatische Verbindung von einem Partiturtext zu einem autorisierten Literaturtext, keine Autorisation eines Partiturtextes durch den Librettisten.

B. Partiturtexte gehören zur Textgenese des Librettos:

6. Wie (3), (4) oder (5), aber stemmatische Verbindung von einem Partiturtext zu einem autorisierten Literaturtext vorhanden.

7. Libretti, bei denen der Komponist Mitautor der Kompositionsvorlage ist, bei denen aber keine stemmatische Verbindung von einem Partiturtext $\mathrm{zu}$ einem autorisierten Literaturtext besteht.

8. Wie (7), aber stemmatische Verbindung von einem Partiturtext zu einem autorisierten Literaturtext vorhanden.

9. Wie (3), (4), (5), (6), (7) oder (8), aber mindestens ein Partiturtext ist durch den Librettisten direkt autorisiert.

10. Libretti von Dichter-Komponisten (Identität zwischen dem hauptsächlichen Autor der Kompositionsvorlage und dem Komponisten).

Es folgen nähere Erläuterungen zu den Kategorien, vor allem zur Verwendung der oben genannten Kriterien, aber auch zu einigen wenigen Beispielen.

Kategorie (1), die unvertonten Libretti, ist hierbei eindeutig. Es sind in diesen Fällen überhaupt keine Partiturtexte vorhanden, und die Grundfrage erübrigt sich somit. $\mathrm{Zu}$ dieser Kategorie können selbstverständlich vollendete Libretti, $^{28}$ aber auch viele

\footnotetext{
${ }^{28}$ Vgl. Jörg Krämer: Deutschsprachiges Musiktheater im späten 18. Jahrhundert. Typologie, Dramaturgie und Anthropologie einer populären Gattung. Teil 2. Tübingen 1998, S. 848-855. Bei vielen Werken fehlt der Komponistenname, bisweilen auch der Librettistenname. Manchmal vermerkt Krämer gesondert: „Offenbar unvertont“. Es ist wahrscheinlich, dass noch mehr Libretti mit fehlendem Komponistennamen im Verzeichnis in
} 
fragmentarische Libretti zählen. Wichtig ist, die nie vertonten Libretti prinzipiell von den Libretti zu unterscheiden, zu denen bloß keine Partiturtexte erhalten, sehr wohl aber erschlossen sind. Diese Libretti sind je nach Entstehungsgeschichte in andere Kategorien einzuordnen.

Die Kategorie (2), Libretti, deren Vertonung nach dem Tod des (hauptsächlichen) Autors erfolgt ist, ist in vielen Fällen fast genau so eindeutig wie die Kategorie (1). Die Partitur des österreichischen Komponisten Wolfgang Florey zur Oper Prärie von 1993 weist vor allem im Bereich der Regiebemerkungen Varianten gegenüber seiner Kompositionsvorlage auf, ${ }^{29}$ Varianten, die aber nicht als Entstehungsvarianten in Bezug auf das Libretto zu bezeichnen sind. Diese Kompositionsvorlage Floreys stammt von Bertolt Brecht und wurde von ihm gerade als Libretto (Brechts Untertitel: „Oper nach Hamsun“) ${ }^{30} 1919$ verfasst; zeit seines Lebens wurde Prärie jedoch weder vertont noch veröffentlicht. Es dürfte evident sein, dass Florey kein Mitautor am Libretto ist und dass Brecht einen mehr als 30 Jahre nach seinem Tod entstandenen Partiturtext nicht autorisiert hat, dieser folglich nicht in die Textgenese dieses Brecht-Werkes gehört.

Offensichtlich etwas problematischer, da im kritischen Bericht der betreffenden Edition besonders unterstrichen, ist der Fall der Arabella von Hugo von Hofmannsthal. Hier ist auf die obige Feststellung zum Unterschied zwischen hauptsächlichem Autor und Mitautor zurückzugreifen. Die kritische Hofmannsthal-Ausgabe schließt aus der Überlieferung die nach dem 15. Juli 1929 entstandenen Materialien deswegen aus, weil Hofmannsthal an diesem Tag gestorben ist. Die später von Richard Strauss überarbeiteten und gedruckten Texte, einschließlich des gedruckten Textbuches und der Notendrucke, sind von Hofmannsthal nicht autorisiert worden. Der Bandbearbeiter Hans-Albrecht Koch schreibt, diese gehören stattdessen zur ,Rezeptionsgeschichte des Werks“ ${ }^{31}$ Nun lässt sich einwenden, dass Strauss, wie der Briefwechsel zeigt, den Text an entscheidenden Punkten schon vor Hofmannsthals Tod beeinflusst hat und deswegen vielleicht als Mitautor gelten könnte Hofmannsthal ist jedoch ohne Zweifel der hauptsächliche Autor, und es erscheint mir wie

\footnotetext{
diese Kategorie einzuordnen sind. Selbstverständlich ist auch aus späterer Zeit eine große Anzahl von unvertonten Libretti vorhanden, wenn auch wohl eher selten veröffentlicht.

${ }^{29}$ Vgl. Wolfgang Florey: Prärie. Oper nach Hamsun von Bertold [sic] Brecht. [Fotokopie einer handschriftlichen Partitur.] Bertolt-Brecht-Archiv, Berlin, Z41/140.

${ }^{30}$ Bertolt Brecht: Prärie. Oper nach Hamsun. In: Ders.: Werke. Große kommentierte Berliner und Frankfurter Ausgabe. Hrsg. von Werner Hecht, Jan Knopf, Werner Mittenzwei, Klaus-Detlef Müller. Bd. 1: Stücke 1. Bearb. von Hermann Kähler. Berlin, Frankfurt/Main 1989, S. 329-341, hier S. 329.

31 Hans-Albrecht Koch: Arabella: Entstehung. Quellen. In: Hugo von Hofmannsthal: Sämtliche Werke. Bd. XXVI: Operndichtungen 4. Hrsg. von Hans-Albrecht Koch. Frankfurt/Main 1976, S. 167-199, hier S. 189 (Kursive im Original).
} 
Koch berechtigt (übrigens auch außerhalb des Kontextes einer HofmannsthalGesamtausgabe), den Schlussstrich der Überlieferung gerade bei Hofmannsthals Tod zu ziehen. Es handelt sich hier um autorschaftsbezogene und autorisationsbezogene Argumente: Der Komponist ist kein hauptsächlicher Autor, und der hauptsächliche Autor (der Librettist) hat die Partiturtexte in keiner Weise autorisiert.

Arabella ist im Unterschied zu Prärie mit einer konkreten Aussicht auf Vertonung entstanden, was allerdings aufgrund des Todes des Librettisten als ein weniger wichtiger Unterschied gelten kann. Die folgende Kategorie (3), Libretti, ohne konkrete Aussicht auf Vertonung entstanden, später vertont, ähnelt in dieser Hinsicht eher Prärie. Sieht man vorläufig vom stemmatischen Kriterium ab, ist, wie ich bereits festgehalten habe, die Mitautorschaft des Komponisten am Libretto eine Bedingung für die Einbeziehung der Partiturtexte. Das heißt, dass Libretti, die ohne Aussicht auf eine konkrete Vertonung geschrieben, unabhängig davon jedoch vertont wurden, wie wir es nicht nur von Metastasio, sondern auch in der deutschsprachigen Librettistik etwa des späten 18. Jahrhunderts ${ }^{32}$ und darüber hinaus kennen, zum linken Teil der Skala gehören. August von Kotzebue brachte 1815 und 1817 in einem zweibändigen Opern-Almanach eine Anzahl von Libretti auf den Büchermarkt und bot sie lesenden Komponisten zur Vertonung an. Der junge Heinrich Marschner folgte der Anregung des Dramatikers relativ schnell und vertonte eines der Libretti, Der Kiffhäuser Berg. Marschners Partitur, obwohl noch zu Lebzeiten Kotzebues entstanden, gehört nicht zur Textgenese des Kotzebue'schen Librettos, da Marschner keineswegs Mitautor des Librettos ist, und auch deswegen, weil Kotzebue es nicht etwa auf dieser Grundlage weiter bearbeitet hat.

In dieser Umgebung ist auch die Existenz von mehrmals vertonten Libretti anzusiedeln, bei denen der Librettist sich an der eventuellen Textbearbeitung des neuen Komponisten nicht beteiligt hat. Dass in solchen Fällen, in erster Linie im 18. Jahrhundert, gelegentlich neue Textbücher gedruckt werden, ist an sich kein Argument für die automatische Einbeziehung der späteren Partiturtexte; es muss vorerst geklärt werden, inwiefern die Textfassung des neuen Textbuches vom Autor der Kompositionsvorlage autorisiert ist oder nicht.

Die scheinbar sehr marginale Differenzierung zwischen (3) und (4), Libretti, ohne konkrete Aussicht auf Vertonung durch den betreffenden Komponisten entstanden,

\footnotetext{
${ }^{32}$ Jörg Krämer stellt für jenen Zeitraum in der „Entwicklung der Librettistik einen Wandel von der in den 1760er Jahren dominierenden direkten Produktion für eine konkrete Aufführung hin zu einer verstärkten Publikation ohne direkten Anlaß auf dem anonymen Markt“" und „ohne konkrete Vertonungsmöglichkeit bzw. -absicht“ fest; Krämer 1998 (Anm. 28), Teil 1, S. 90.
} 
später vertont, ist an Franz Grillparzers Melusina anschaulich zu machen. Dieses Libretto war sehr wohl für einen Komponisten, Ludwig van Beethoven, vorgesehen, und die Vertonung war auch mit ihm vereinbart, eine Vertonung jedoch, die von Beethoven dann doch nie ausgeführt wurde. Später vertonte Conradin Kreutzer den Text; der entscheidende Punkt ist, dass dies ganz ohne Zusammenarbeit mit Grillparzer und auch ohne dessen Autorisation geschehen ist. ${ }^{33}$ Kreutzer ist daher kein Mitautor, und obwohl Grillparzers Text, und zwar in der von ihm ursprünglich für Beethoven vorgesehenen Fassung, erst im Kontext der Kreutzer'schen Vertonung gedruckt wurde, sollte Kreutzers Partitur in einer Edition von Grillparzers Melusina nicht zur Überlieferung gezählt werden. Der Partiturtext wäre hier nicht sinnvoll als Teil der Textgenese des Librettos $\mathrm{zu}$ beschreiben. ${ }^{34}$ Die im Partiturtext enthaltenen Varianten gehören der Werkrezeption an. ${ }^{35}$ Die Kriterien sind dieselben wie bei der Kategorie (3).

Noch zur Gruppe A, in der Partiturtexte der Textgenese des Librettos nicht zugerechnet werden sollten, gehört die Kategorie (5), Libretti, deren Vertonung zwischen Librettist und Komponist vereinbart ist, bei denen der Komponist jedoch nicht als Mitautor am Text gelten kann. Offensichtlich ist dies der Fall bei Eduard Mörikes Libretto Die Regenbrüder (1834-1837 entstanden, mit Hermann Kurz als Mitautor); der Komponist Ignaz Lachner hat Mörike zuerst um ein Libretto gebeten, scheint aber nicht an der Kompositionsvorlage als Autor oder Mitautor beteiligt gewesen zu sein; er drängt auf die Vollendung des Librettos (die Mörike dann wegen Krankheit an Hermann Kurz überträgt), schlägt auch ,in musikal. Hinsicht noch hie u. da kleine Veränderungen in meinen [Mörikes] Versen“36 vor, und kommt dann während der Kompositionsarbeit mit „,sehr MODEST[en]“ Forderungen an Mörike, der allerdings daraufhin „ihm auch diese vollends ausgeredet“

\footnotetext{
${ }^{33}$ Kreutzer verhandelte ausschließlich mit Grillparzers Verleger. Vgl. zur ganzen Entstehungsgeschichte dieses Librettos Reinhold Backmanns Darstellung in Franz Grillparzer: Sämtliche Werke. Historisch-kritische Gesamtausgabe. Mit Unterstützung des Ministeriums für innere und kulturelle Angelegenheiten im Auftrage der Stadt Wien hrsg. von August Sauer (†), fortgeführt von Reinhold Backmann. Abt. 1, Bd. 19. Wien 1939, S. 351.

${ }^{34}$ Vgl. Backmann 1939 (Anm. 33), S. 57-60.

${ }^{35}$ Reinhold Backmanns Lösung innerhalb der Grillparzer-Ausgabe war eine andere. Auch er nimmt die Partitur Kreutzers zu Recht nicht auf; dagegen verzeichnet er die handschriftlichen Änderungen Kreutzers in einer Abschrift von Grillparzers Text. Das zu Kreutzers Werk gehörige Material enthält einen (als Stellvertreter) für Grillparzers Werk relevanten Textträger: Die früheste von Kreutzer benutzte Abschrift ist zweifellos auch für die Überlieferung des Librettos interessant. Dann führt Backmann aber auch im Detail Notizen und Änderungen Kreutzers in weiteren Abschriften (Literaturtexten) an, was m.E. nicht berechtigt ist, will man nicht auch der Rezeption des Librettos im Detail nachgehen.

${ }^{36}$ Brief von Eduard Mörike an Hermann Kurz, 26. Mai 1837. In: Eduard Mörike: Werke und Briefe. Bd. 12: Briefe 1833-1838. Hrsg. von Hans-Ulrich Simon. Stuttgart 1986, S. 91f., hier S. 92.
} 
habe. ${ }^{37}$ Wie dem auch sei, Lachners direkter, tatsächlicher Einfluss auf die Textgestalt des Literaturtextes ist insgesamt als sehr gering einzustufen.

Wenn nur eine Vereinbarung zwischen Komponist und Librettist besteht, der Komponist sich aber nicht oder nur am Rande an der Textentstehung beteiligt und daher nicht als Mitautor gezählt werden kann, erscheint es mir nicht angebracht, die Partiturtexte anzuführen. Anders ausgedrückt: Ist der Komponist nur Textempfänger und Textbearbeiter, hat er keinen beachtlichen Einfluss auf die Ausgestaltung der Kompositionsvorlage ausgeübt, sind die Partiturtexte nicht sinnvoll der Textgenese zuzurechnen, es sei denn, der Librettist hat nachträglich den Literaturtext nach einem Partiturtext revidiert. ${ }^{38}$

Gerade bei diesem letzteren Sonderfall - stemmatische Abhängigkeit eines autorisierten Literaturtextes von einem Partiturtext - scheint mir die Grenzlinie zwischen der kleineren Gruppe A (bei der die Partiturtexte nicht zur Textgenese des Librettos gehören) und der größeren Gruppe B (bei der dies dagegen der Fall ist) zu verlaufen. Zu Letzterer gehört die Kategorie (6), bei der der Komponist immer noch kein Mitautor, aber eine stemmatische Verbindung von einem Partiturtext zu einem autorisierten Literaturtext feststellbar ist. Dieser Fall scheint beispielsweise beim 1991 oder 1992 als Kompositionsvorlage fertiggestellten ${ }^{39}$ Gesualdo von Richard Bletschacher vorzuliegen; an sich skeptisch gegenüber den Kürzungen, die der Komponist Alfred Schnittke an Bletschachers Vorlagentext vornahm, revidiert Bletschacher für eine viel spätere Literaturtextausgabe seinen Text gerade von Schnittkes Partitur ausgehend, ,um keine Differenzen zur Partitur aufscheinen zu lassen“. ${ }^{40}$ Hier gehört Schnittkes Partitur wiederum zur Überlieferung, und der dort enthaltene Text zur Textgenese, was ohne Bletschachers Revision nicht unbedingt der Fall wäre.

Von den Kategorien (7) und (8) dürfte besonders (8) die allermeisten Opernlibretti, zumindest die meisten aus dem 19., 20. und dem 21. Jahrhundert umfassen. Hier handelt es sich um Libretti, bei denen der Komponist Mitautor der Kompositionsvorlage ist. Der Unterschied zwischen den beiden Kategorien liegt im stemmatischen Kriterium, das

\footnotetext{
37 Mit oben genannter Ausnahme zitiert und referiert nach Albrecht Bergolds Kommentar zur Entstehungsgeschichte der Regenbrüder in Eduard Mörike: Werke und Briefe. Bd. 7: Idylle vom Bodensee. Dramatische Schriften. Vermischte Schriften. Hrsg. von Albrecht Bergold. Stuttgart 2008, S. 469-477, hier S. 470.

38 Dass Bergold trotzdem die erhaltene Partitur zur Oper Lachners als Teil der Überlieferung anführt und Lesarten aus ihr verzeichnet (Bergold in Mörike 2008, Anm. 37, S. 483ff.), lässt sich aus einem anderen Grund rechtfertigen: Sowohl die Textgrundlage, ein gedruckter Literaturtext, als auch die Partitur gehen auf eine verschollene Handschrift zurück und zeugen somit indirekt von dieser früheren Stufe der (eigentlichen) Textgenese. Die Partitur wäre m.E. als Ersatz, als Stellvertreter eher denn als Teil der eigentlichen Überlieferung zu betrachten.

${ }^{39}$ Richard Bletschacher: Essays zu Musik und Musiktheater. Wien, Köln, Weimar 2008, S. $241 \mathrm{f}$.

${ }^{40}$ Richard Bletschacher: Vorbemerkung. In: Ders.: Theaterstücke für Musik. Wien, Köln, Weimar 2011, S. 7-9, hier S. 9. Vgl. zur Entstehungsgeschichte von Libretto und Oper Bletschacher 2008 (Anm. 39), S. 230-254.
} 
aber in diesem Fall belanglos für die übergeordnete Einstufung der beiden Kategorien ist: Bei beiden sollen die Partiturtexte vor allem aufgrund des autorschaftsbezogenen Kriteriums zur Textgenese und die entsprechenden Textträger zur Überlieferung gerechnet werden.

Genau wie bei Kategorie (5) geht es bei (7) und (8) um die Bestimmung einer Mitautorschaft. Es dürfte sich hier um eine Zusammenarbeit handeln, in der sich Anteile des Komponisten am Wortmaterial oder an der Struktur der Kompositionsvorlage konkret feststellen lassen. Das dürfte die meisten Libretti betreffen, die der Zusammenarbeit Hofmannsthal/Strauss entsprungen sind, ebenfalls einige der Bühnenwerke von Brecht und Weill, es betrifft ebenfalls eine große Anzahl bekannter Beispiele wie Stephanies und Mozarts Die Entführung aus dem Serail, Fouqués und Hoffmanns Undine oder Die lustigen Weiber von Windsor von Mosenthal und Nicolai.

Die Kategorie (9) bezieht sich auf den Fall, in dem der Librettist (der hier, im Unterschied zur folgenden Kategorie, mit dem Komponisten nicht identisch ist) einen Partiturtext gesondert autorisiert hat. Hier muss allerdings auch die schwierige Frage nach einer Autorisation ,als was' mit einbezogen werden: als Vorlage für die Bühnenumsetzung in Verbindung mit der Musik oder auch davon unabhängig als Text ohne musikalische Notation?

Die Kategorie am rechten Ende der Skala, (10), vertonte Libretti von DichterKomponisten, wäre theoretisch gesehen eindeutig: Sowohl das autorschafts- als auch das autorisationsbezogene Kriterium wären durch die Identität von Librettist und Komponist von vorneherein erfüllt.

Allerdings ist hier in gewissen Fällen eine weitere Sichtweise möglich. Cristina Urchueguía bechreibt in Bezug auf Lohengrin und Der fliegende Holländer das Verhältnis zwischen Literaturtexten und Partiturtexten, zwischen Libretto und Vertonung wie folgt: „Wagner verfaßte eine Vorlage; als diese vollendet war, entstanden davon ausgehend zwei unabhängige Werke: eine Oper und eine Dichtung, die den Stoff, die dramaturgische Anlage und viel Wortlaut miteinander teilen“. ${ }^{41}$ Zwei eigenständige Zielformen führen bei Urchueguía zu einer Einteilung in zwei separate Werke. Auch der beachtenswerte Aspekt der Autorisation ,als was' wird hierdurch aktualisiert. Urchueguías Kriterien unterscheiden sich also von denen in meiner Auslegung und bieten in ihrer Radikalität eine wichtige Differenzierung, aber hervorheben ist insbesondere, dass auch bei ihr das stemmatische Kriterium für die Abgrenzung eines Werks und die Einbeziehung der Partiturtextträger in die Überlieferung des Librettos von großer Bedeutung ist.

\footnotetext{
${ }^{41}$ Urchueguía 2004 (Anm. 15), S. 298.
} 
Es soll übrigens auch vermerkt werden, dass von Komponisten eingerichtete, aber zum großen Teil wörtlich übernommene Sprechdramentexte - also ein großer Teil der nicht ganz glücklich so bezeichneten ,Literaturopern“ oder ,Literaturlibretti ${ }^{42}$ in der Tradition von Debussy (Pelléas et Mélisande) und Strauss (Salome und Elektra) - sinnvoll zu dieser Kategorie 10 geordnet werden sollen. ${ }^{43}$

Der Entstehungsprozess ist in jedem Einzelfall genau zu prüfen, um die Zuordnung zu einer bestimmten Kategorie vornehmen zu können; dies wäre jedoch auch unabhängig von dieser Problematik wegen der zentralen Kriterien der Autorschaft, Autorisation und Stemmatik notwendig gewesen. Die zehn Kategorien sind dabei kein Selbstzweck, sondern als ein Werkzeug zu verstehen, um die Frage nach dem Stellenwert von Partiturtexten in der Textgenese eines Librettos beantworten $\mathrm{zu}$ können und um die Abgrenzung einer Überlieferung in solchen Fällen auch editionstheoretisch besser fassen zu können.

\begin{abstract}
This essay deals with one of the main questions in a future Germanistic editorial theory of the opera libretto as a literary work: under which circumstances are texts contained in musical scores to be regarded parts of the genesis of the libretto and these text carriers to be seen as parts of its transmission? In this essay, it is argued that the answer to this question is dependent on the individual development process of the libretto and decided by three criteria: an author-oriented, an authorization-oriented and a stemmatic one. A preliminary classification of development processes of librettos, based upon these criteria, is sketched.
\end{abstract}

\footnotetext{
${ }^{42}$ Der Terminus Literatur-Libretto zuvor bei Thomas Beck: Bedingungen librettistischen Schreibens: Die Libretti Ingeborg Bachmanns für Hans Werner Henze. Würzburg 1997, S. 57.

${ }^{43}$ Die Kategorisierung ist insgesamt mit den bei Karin Hochradl angeführten „,[d]rei optionale[n] Formen von Libretti“ bzw. deren Entstehungsgeschichten schwer kompatibel, zumal Hochradl implizit von fertigen Opern ausgeht. Die „Personalunion von Komponist und Librettist“ ist in der Kategorie 10 enthalten, „exemplarische Librettistik als Zusammenarbeit von Dichter und Komponist" entspricht am ehesten den Kategorien 7 und 8 während „die selbständige Aneignung eines literarischen Werkes“ (durch einen Komponisten!) streng genommen in den Kategorien 2, 3, 4, 5, 6 (wenn man dabei Libretti als literarische Werke betrachtet) und auch 10 (Hochradl bezieht sich im Kontext gerade auf die ,Literaturoper') vorkommen könnte; vgl. Hochradl 2010 (Anm. 27), S. 37.
} 
